\title{
ILCEA
}

Revue de l'Institut des langues et cultures

d'Europe, Amérique, Afrique, Asie et Australie

$45 \mid 2022$

Femme(s) et sorcellerie en Espagne et en Italie à l'époque moderne

\section{Le Tractatus de Strigiis de Bernard Rategno de Côme (c. 1510) : le regard d'un inquisiteur sur la sorcellerie et démonologie du nord de l'Italie}

The Tractatus de Strigiis by Bernard Rategno of Como (c. 1510): An Inquisitor's View of Witchcraft and Demonology in Northern Italy

\section{Alessia Belli}

\section{OpenEdition}

\section{Journals}

Édition électronique

URL : https://journals.openedition.org/ilcea/14352

DOI : 10.4000/ilcea.14352

ISSN : 2101-0609

Éditeur

UGA Éditions/Université Grenoble Alpes

\section{Édition imprimée}

ISBN : 978-2-37747-330-4

ISSN : 1639-6073

Référence électronique

Alessia Belli, «Le Tractatus de Strigiis de Bernard Rategno de Côme (c. 1510) : le regard d'un inquisiteur sur la sorcellerie et démonologie du nord de l'Italie », ILCEA [En ligne], 45 | 2022, mis en ligne le 31 janvier 2022, consulté le 31 janvier 2022. URL : http://journals.openedition.org/ilcea/14352 ; DOI : https://doi.org/10.4000/ilcea.14352

Ce document a été généré automatiquement le 31 janvier 2022.

(c) ILCEA 


\section{Le Tractatus de Strigiis de Bernard Rategno de Côme (c. 1510) : le regard d'un inquisiteur sur la sorcellerie et démonologie du nord de l'Italie}

The Tractatus de Strigiis by Bernard Rategno of Como (c. 1510): An Inquisitor's View of Witchcraft and Demonology in Northern Italy

\section{Alessia Belli}

Bernard Rategno, inquisiteur dominicain actif dans le diocèse de Côme, dans le nord de l'Italie, rédige au début $\mathrm{du} \mathrm{xvI}^{\mathrm{e}}$ siècle un petit opuscule, connu sous le nom de Tractatus de Strigiis ${ }^{1}$. Il s'agit d'un traité qui s'adresse aux inquisiteurs, pour tenter d'expliquer les actions diaboliques de la secte des striges composée tant d'hommes que de femmes. Ces individus participeraient la nuit au « jeu de la bonne société » (ludus bonce societatis), en entretenant des rapports avec le diable, qui leur apparaît sous forme humaine. Ils pratiquent ensuite plusieurs actes ignobles.

\section{L'auteur}

2 Né très probablement aux alentours de la moitié du $\mathrm{xv}^{\mathrm{e}}$ siècle dans le diocèse de Côme, appartenant à la province de saint Pierre Martyr, Bernard Rategno fait partie de la Congrégation observante dominicaine de Lombardie (Tavuzzi, $2007: 220$ ). Après avoir étudié la théologie à l'Université de Pavie, nous le retrouvons en 1474 au Sacré Palais de Rome, en tant que lecteur des Sentences (Lavenia, 2010 : 554). La carrière de Rategno est ensuite partagée entre la prédication et l'enseignement académique, après avoir obtenu par le Chapitre général de l'ordre des Prêcheurs la possibilité de recouvrer la fonction de lector dans toutes les universités ${ }^{2}$. Parallèlement, il est à la tête de plusieurs 
couvents dominicains situés dans l'Italie septentrionale, à savoir: le couvent San Giovanni Pedemonte de Côme (1490) ; le couvent dominicain de Modène (1493-1494) ; le couvent Sant'Andrea de Faenza (1494-1496) et le couvent dominicain de Crémone (1501-1502) (Giorgetta, 2003: 28; Lavenia, 2010 : 554; Tavuzzi : 220). L'année 1505 représente un tournant décisif pour la carrière de Bernard Rategno. En effet, le 20 mai de cette même année, il est nommé inquisiteur du diocèse de Côme ${ }^{3}$, sous le pontificat de Jules II (1503-1513). L'activité inquisitoriale de Bernard de Côme se concentre surtout en Valteline et Valchiavenna, deux vallées situées dans la partie septentrionale dudit diocèse. Malheureusement, une grande partie de la documentation archivistique du tribunal de l'Inquisition de ces régions a été détruite ou a été perdue (Giorgetta, 2007 : 97) ; nous pouvons néanmoins estimer que les persécutions inquisitoriales contre les sorciers et les sorcières dans ces territoires se sont concentrées entre la troisième décennie $d u x^{e}$ siècle et 1512 . Date à laquelle la république des Ligues rhétiques ${ }^{4}$ s'empare du contrôle politico-religieux de la Valteline et de la Valchiavenna, au détriment du duché de Milan qui dominait ces lieux à cette époque. La république des Trois Ligues procède ensuite à l'expulsion des inquisiteurs, en poursuivant toutefois elle-même la répression de la sorcellerie diabolique (Belli \& Estuardo Flaction, 2019 : 28-29).

3 Si nous revenons maintenant à Rategno, nous pouvons le situer en Valchiavenna durant l'été 1506, lorsque le Conseil général de ce lieu convoque l'inquisiteur afin de lutter contre le crime d'hérésie ${ }^{5}$; nous n'avons pas d'autres traces documentaires de l'activité juridique de Rategno (Duni, 2013a : 354). Bernard de Côme occupe la fonction d'inquisiteur jusqu'à sa mort, survenue probablement entre 1511 et 1515 (Lavenia, $2010: 555)$.

\section{L'œuvre}

Pendant son activité inquisitoriale, Bernard Rategno rédige deux œuvres ayant comme thématique principale la répression de l'hérésie et de la sorcellerie. La première, la Lucerna inquisitorum horetice pravitatis est un véritable manuel pensé pour les inquisiteurs. Rategno présente, sous forme d'articles suivant un ordre alphabétique, tous les éléments utiles pour mener correctement les procès contre les hérétiques. Le deuxième écrit, le Tractatus de Strigiis ${ }^{6}$, est introduit par Rategno dans un des articles de la Lucerna ${ }^{7}$. Dans le De Strigiis, Bernard de Côme développe la question de la secte des striges (secta strigiarum), et met en lumière, toujours au bénéfice de ses collègues inquisiteurs, les composantes qui définissent la secte des sorciers et sorcières. Celle-ci serait en train de s'implanter toujours plus dans les régions du nord de l'Italie. Les deux œuvres seront publiées ensemble pour la première fois à Milan en 1566 (Belli \& Estuardo Flaction, 2019:7). Il faut souligner que, dans l'état actuel de la recherche, aucune version manuscrite du De Strigiis n'a été retrouvée. De même, la datation exacte de ce traité reste incertaine. Grâce à quelques éléments de contenu présents dans le texte, nous pouvons affirmer qu'il aurait été rédigé entre 1505 et $1510^{8}$. Comme Rategno a été nommé inquisiteur en 1505, nous pouvons estimer qu'il a écrit ses manuels quelques années après le début de son activité inquisitoriale, soit vers 1510 . Cela signifie que la première édition de Milan daterait d'environ 55 ans après la rédaction originelle du traité. Étant donné que nous n'avons pas à disposition de versions manuscrites antérieures, nous ne pouvons pas déterminer si, ni à quel niveau, 
le texte a subi des modifications. Après l'édition de Milan de 1566, suivent cinq autres, publiées entre 1584 et 1669, que nous décrivons ici brièvement (ibid. : 255-259).

5 La première édition a été publiée à Milan en 1566 par les frères Metios, sur demande d'Angelo Zampa, inquisiteur de Milan, et ancien vicaire de l'inquisiteur dominicain de Côme, Michele Antonio Ghislieri (Lavenia, 2010: 555). Ce dernier avait été désigné en 1551 commissaire général de l'Inquisition; il devient pape, sous le nom de Pie V, en 1566. Ceci dit, une cinquantaine d'années après sa rédaction, l'œuvre de Rategno attire l'attention de l'entourage d'un des papes les plus actifs dans le développement de la politique inquisitoriale. En effet, le pontife, après avoir renouvelé l'Index des livres interdits, arrive à éradiquer une grande partie des hérésies du sol italien (protestants et spirituels, notamment) (Belli \& Estuardo Flaction, 2019: 255). Nous pouvons nous demander si le choix de publier le traité de Rategno n'est pas un hommage que Ghislieri fait à son prédécesseur.

6 En 1584, Francisco Peña, canoniste aragonais de la curie romaine, fait publier à Rome par Bartolomeo Graffi une deuxième édition commentée par ses soins du Tractatus de Strigiis. La figure de Peña n'est pas sans intérêt. Plus précisément, le canoniste avait déjà réédité, commenté et publié plusieurs textes sur les hérésies, ainsi que quelques manuels inquisitoriaux. Parmi ces titres on trouve le célèbre Directorium inquisitorum (1376) de Nicolas Eymerich (Duni, 2013b : 306). Pourquoi Peña aurait-il jugé nécessaire de rééditer ces traités ? D’après Duni (2013b), la génération des inquisiteurs de la deuxième moitié du Xvi ${ }^{e}$ siècle, après avoir éradiqué la présence protestante du territoire italien, s'oriente à nouveau vers la répression des hérésies et de la sorcellerie diabolique. Il était alors judicieux pour les juristes - tels que Peña - d'offrir aux inquisiteurs une procédure mise à jour et surtout cohérente avec les volontés du SaintOffice. Ce dernier était, quant à lui, plus réticent à se lancer dans une nouvelle chasse aux sorcières (Lavenia, 2010:555). En rééditant entre autres l'œuvre de Rategno, le canoniste aragonais s'efforce de répondre à ces enjeux (Belli \& Estuardo Flaction, 2019 : 256).

7 La troisième édition sans les commentaires de Peña voit le jour à Venise en 1584. Cette version est insérée dans l'immense œuvre Tractatus universi iuris, le recueil de traités juridiques voulu par le pape Grégoire XIII (1572-1585).

8 La quatrième édition, également publiée à Venise en 1596 par Marco Antonio Zaltieri, est simplement une réédition de la publication de Rome de 1584 .

9 Avec les deux dernières éditions, nous changeons de zone géographique et de siècle. En effet, la version du Tractatus de Strigiis avec les commentaires de Peña est publiée à Lyon en 1620 et 1669 dans les recueils des traités démonologiques qui accompagnaient le Malleus Maleficarum (1487) d'Institoris (Belli \& Estuardo Flaction, 2019 : 258).

10 Nous pouvons affirmer alors que le petit traité de Bernard de Côme, d'à peine 12 folios pour la publication de Milan, a connu une certaine circulation, qui dépasse le seul territoire de la péninsule italienne.

11 Le traité peut être divisé en trois grandes parties. La première correspond à la partie introductive, où Rategno dresse le portrait de la secte des striges active dans le nord de l'Italie. Ensuite, l'inquisiteur de Côme entre dans le cœur du débat concernant la réalité - ou l'irréalité - du vol nocturne des sorciers et sorcières et de leurs assemblées avec le diable. Dans ce segment, il est également question pour l'auteur d'aborder en profondeur les enjeux du canon Episcopi. Le traité s'achève avec la partie la plus 
novatrice du traité, où Rategno expose la procédure juridique à suivre par les inquisiteurs, afin de mener leurs enquêtes à l'encontre des membres de cette secte (Belli \& Estuardo Flaction, 2019 : 299).

\section{La secta strigiarum et la cérémonie d'initiation} devront faire face à une secte composée d'hommes et de femmes (surtout de ces dernières), qui depuis plusieurs années est en train de s'enraciner toujours plus dans les territoires septentrionaux italiens. Les membres de ce groupe auraient des liaisons avec le diable. Nous pouvons mettre en lumière le fait que Rategno souligne la présence d'hommes; cela est particulièrement intéressant car, comme nous le savons, la tradition de la sorcellerie italienne est une histoire plutôt féminine. D'ailleurs en Italie, c'est la femme qui d'habitude est "sorcière ", alors que dans d'autres territoires - tels que la Suisse romande actuelle, par exemple - l'on retrouve plusieurs cas de sorciers hommes ${ }^{10}$. Il faut néanmoins se poser la question si la mention des hommes faite par Rategno ne ferait pas partie d'un formulaire typique des traités de sorcellerie.

L'inquisiteur de Côme se lance ensuite dans une explication étymologique un peu maladroite du mot strigia, utilisé en Lombardie pour désigner les membres de cette secte diabolique. D'après l'auteur, ce terme aurait alors deux étymologies possibles : la première propose que ce mot tirerait son origine du nom du Styx, le fleuve infernal de la mythologie gréco-romaine. Ce mot signifierait en effet, d'après Rategno, « enfer ». Les striges seraient ainsi assimilées au domaine infernal. La deuxième étymologie proposée par l'inquisiteur remonterait au terme grec de stigetos ( $\sigma \tau \rho u ́ \gamma \varepsilon v v$ ), qui serait la tristitia latine. Les membres de la secta strigiarum seraient alors la cause du malheur des personnes, dû à la propagation de leurs différents maléfices. Toutefois, les propositions étymologiques de Rategno sont fausses d'un point de vue linguistique (Belli \& Estuardo Flaction, 2019 : 300-301). Il est acquis pour l'époque de Rategno que l'origine correcte du terme «strige » était déjà connue. En effet, deux démonologues espagnols du milieu $\mathrm{du} \mathrm{XVI} \mathrm{I}^{\mathrm{e}}$ siècle, Alphonse de Castro, théologien franciscain, et Diego Simancas, juriste et inquisiteur, l'exposent clairement dans leurs traités respectifs ${ }^{11}$ : le mot "strige » est dérivé du nom latin de la strix (strige au pluriel), créature de la mythologie latine, mi-femme, mi-rapace, «qui passait chez les Anciens pour sucer le sang des enfants » (Gaffiot, 1934: 1484) ${ }^{12}$. Il faut alors se demander pour quelle raison Rategno aurait décidé de contourner cette étymologie, en en proposant deux autres. Ignorait-il vraiment l'origine de ce terme? Ou d'autres raisons peuvent-elles être évoquées?

Rategno est ensuite particulièrement exhaustif sur les étapes qui caractérisent la cérémonie d'initiation ${ }^{13}$.

Tout d'abord, celle-ci a lieu la nuit du jeudi au vendredi, lorsque les striges rencontrent le diable, près des villages et des bourgs. Dans le paragraphe introductif, ces assemblées sont appelées «ludum bonce societatis » et le diable s'y manifeste sous forme humaine. Afin d'appartenir complètement à cette «bonne société », les futures striges doivent rejeter la foi chrétienne, le baptême, Dieu, ainsi que la Vierge et piétiner une croix tracée par terre. Après quoi, ces individus sont prêts à se soumettre totalement au diable, en lui prêtant serment de fidélité et d'obéissance en mettant leurs mains dans celles de leur nouveau maître. Finalement, le diable est leur seul seigneur et devra être considéré comme leur unique dieu. 

d'initiation du ludus font partie d'une longue tradition d'accusations qui ne sont pas uniquement liées au domaine de la sorcellerie diabolique. En effet, l'apostasie et les actes blasphématoires étaient déjà largement utilisés dans les siècles précédents pour les imputations à l'encontre des hérétiques ${ }^{14}$. De plus, la cérémonie d'initiation décrite par Rategno suit parfaitement la tradition des traités de démonologie rédigés par des théologiens dominicains à partir du milieu du xve siècle, au nord et au sud des Alpes. Nous faisons ici référence, par exemple, au traité moralisateur Formicarius de Jean Nider ${ }^{15}$ ou aux écrits du Milanais Girolamo Visconti, rédigés environ en $1460^{16}$. Cette cérémonie, caractérisée par la forte présence des éléments d'apostasie et de blasphème, évoque ainsi pour le nouveau membre de la secte des striges, d'abord un moment de scission avec son identité chrétienne, puis un processus de soumission totale au nouveau maître, le diable (Chène, Ostorero, Paravicini Bagliani \& Utz Tremp, 1999 : 243). Plus spécifiquement, il apparaît assez évident que les démonologues construisent ce rituel particulier en détournant et en diabolisant les éléments centraux de la messe chrétienne ou d'autre cérémonies laïques (l'hommage vassalique, par exemple ${ }^{17}$ ).

De même, lors des assemblées, ces hommes et femmes diaboliques entretiendraient des rapports sexuels avec le diable, qui apparaît sous forme humaine, succube pour les hommes et incubes pour les femmes ${ }^{18}$. Ces êtres, issus de la tradition de la mythologie latine, et largement repris par les penseurs scolastiques ainsi que par les démonologues, sont des démons qui utilisent les rapports charnels pour soumettre les hommes et les femmes à leur volonté, en les souillant de leur luxure (Belli \& Estuardo Flaction, 2019 : 317).

18 Après quoi, il faut souligner que Rategno, une fois achevée la description de la cérémonie d'initiation, ne s'étend pas en profondeur sur les détails maléfiques pratiqués par les striges lors du ludus. L'intention principale du De Strigiis de Bernard de Côme n'est pas alors de donner un portrait exhaustif des procédés des striges. Quel est donc le but principal de cette œuvre?

\section{La réalité du ludus}

Afin de répondre à cette question, nous devons passer à la partie centrale du traité de notre inquisiteur dominicain. Pour Rategno, il est question d'aborder le débat sur la réalité ou l'irréalité du ludus. Sa position est claire dès le début : en effet, il répète sans cesse tout au long de ses pages que les striges se rendent physiquement aux rencontres avec le diable, en état de veille et conscientes de leurs actions ${ }^{19}$. D'après l'auteur, il y a deux façons pour ces individus de rejoindre les lieux des rencontres. Si la localité est proche, ils s'y rendent à pied, autrement ils y sont amenés directement par le diable (Belli \& Estuardo Flaction, 2019: 268). Ensuite, Rategno fait allusion aux procès qu'il avait menés auparavant ${ }^{20}$. Il tient à préciser que les déplacements dans les airs, ainsi que tout ce que ces personnes ont avoué avoir commis lors des assemblées - les actes apostats et les rapports charnels avec le diable - ont vraiment eu lieu. D'ailleurs, il utilise souvent l'expression " corporaliter et realiter, ac veraciter " pour définir tout ce que les striges font (ibid.). Voilà donc dévoilé le véritable but du De Strigiis de Bernard Rategno: il s'agit pour l'inquisiteur de Côme de démontrer que les pratiques des membres de la secte des striges ont vraiment lieu et ne sont pas le fruit de 
manipulations diaboliques. En proposant ce type de discours, Bernard de Côme s'inscrit parfaitement dans le débat démonologique de la fin $\mathrm{du} \mathrm{xv}^{\mathrm{e}}$ siècle. Ce dernier se développe sans cesse sur le réalisme du vol et des accusations mobilisées contre les sorciers et les sorcières.

Afin d'apporter de la matière à son propos, Rategno utilise plusieurs cas de figure provenant de sa propre expérience en tant qu'inquisiteur dans le diocèse de Côme ; il se fait alors fort de son autorité juridique pour prendre place dans cette querelle, qui voit s'opposer plusieurs savants laïcs et ecclésiastiques de l'époque. Par exemple, Rategno affirme que, comme toutes les personnes accusées (hommes et femmes), dans toute l'Italie, avouent avoir commis les mêmes faits lors du ludus, ces actions doivent être forcément vraies ${ }^{21}$. Ou encore, notre auteur soutient qu'il peut arriver que, au moment du transport par le diable des striges aux assemblées, elles soient, par volonté divine, laissées à elles-mêmes pendant le trajet. Elles sont ensuite vues à plusieurs kilomètres de leur domicile. D'après l'inquisiteur de Côme, la seule explication pour ce fait est que ces événements adviennent réellement et qu'ils ne sont pas des illusions diaboliques ou de simples rêves ${ }^{22}$. Rategno soutient encore que, comme beaucoup de membres de la secte des striges sont depuis plusieurs temps remis par les inquisiteurs au bras séculier pour être condamnés au bûcher, le Saint-Siège ne pourrait jamais accepter que des personnes soient exécutées sur la base de faits qui auraient lieu uniquement par illusion ou en rêve ${ }^{23}$.

Or, pourquoi Bernard Rategno a-t-il besoin d'affirmer constamment que les rencontres diaboliques, ainsi que les pratiques des striges se produisent vraiment? En effet, l'inquisiteur de Côme se positionne explicitement contre ceux qui - il ne dévoile pas de noms - utilisent le célèbre canon Episcopi pour soutenir la dimension illusoire des pratiques des sorciers et sorcières ${ }^{24}$.

Ce petit texte, dont la version la plus ancienne remonte aux Causes synodales de l'abbé allemand Réginon de Prüm (env. 906), fut intégré par Gratien et son école juridique dans leur Décret autour de la moitié du XII ${ }^{\mathrm{e}}$ siècle, le rendant ainsi partie intégrante du Corpus iuris canonici (Ostorero, 2011: 574-575). Le canon est le premier à décrire la croyance au cortège nocturne de Diane: la nuit, des femmes scélérates seraient convaincues de chevaucher des animaux en compagnie de la déesse. Ces femmes se soumettraient à elle, en l'adorant comme leur maîtresse. Le canon Episcopi considère alors que :

[...] Une foule innombrable de gens, trompés par cette fausse opinion, croient que ces choses sont vraies et, se détournant de la vraie foi et tombant dans l'erreur des païens, pensent qu'il existe d'autres divinités ou déités en dehors du Dieu unique. C'est pourquoi les prêtres [...] doivent prêcher avec insistance au peuple de Dieu pour qu'il sache que toutes ces choses sont tout à fait fausses, inspirées non par l'esprit de Dieu, mais par un mauvais esprit qui provoque de telles illusions dans la pensée des fidèles. (Ostorero, 2011 : 573)

Donc, d'après le canon Episcopi, croire que des femmes pourraient s'en aller en compagnie de la déesse Diane équivaudrait au péché d'infidélité (Belli \& Estuardo Flaction, 2019 : 166). Il faut comprendre comment Rategno articule son discours pour ne pas démentir ouvertement une loi issue du droit canon.

Pour ce faire, l'inquisiteur de Côme dispose de plusieurs stratagèmes. Tout d'abord, la question serait relativement facile à résoudre : tout simplement, le canon Episcopi ne s'exprimerait pas sur la secte des striges dont il est question à l'époque de Rategno. 
En effet, pour ce dernier, la secte aurait commencé à se manifester vers 1360 , soit plusieurs siècles après les faits décrits par le canon Episcopi, que Rategno fait remonter faussement au IV ${ }^{\mathrm{e}}$ siècle (Belli \& Estuardo Flaction, 2019 : 273-274). De ce fait, les propos de l'auteur du DeStrigiis n'entreraient pas en conflit avec le droit ecclésiastique : les femmes du canon Episcopi et les striges sont deux choses différentes. Les premières peuvent tout à fait être une illusion démoniaque ; les deuxièmes sont par contre une réalité (ibid. : 339).

Nous pouvons citer un autre exemple, dans lequel l'inquisiteur de Côme réfléchit par l'absurde ${ }^{25}$. Il admet que le canon Episcopi pourrait également concerner les striges. Il affirme toutefois que, si nous nous penchons sur le contenu du texte législatif, nous noterons que le canon Episcopi affirme bel et bien que les déplacements des sorcières sont des illusions diaboliques, mais il ne les désigne jamais comme impossibles. Le texte $\mathrm{du}$ Corpus iuris canonici ne nie pas ouvertement la possibilité que certains individus puissent se rendre aux assemblées diaboliques physiquement; d'ailleurs, il ne réfute pas clairement que ces personnes puissent être amenées par le diable au lieu du ludus. Alors, pour résumer, le canon Episcopi en n'énonçant pas précisément que le vol nocturne des sorciers et des sorcières est impossible, le rend possible (Belli \& Estuardo Flaction, 2019: 342). Cette démarche suit parfaitement celle qui est proposée par Girolamo Visconti, démonologue dominicain de Milan, dans son œuvre Lamiarum sive striarum opusculum (env.1460). Dans les pages de son texte, l'auteur propose un exemple plus concret qui pourrait faciliter la compréhension du raisonnement: Visconti formule que le duc de Milan "pourrait être roi d'Italie ou empereur, mais pourtant il ne l'est pas. Il est possible qu'il le soit mais cela est faux» (ibid.: 80-81). Le manque de précision du célèbre canon permet à Visconti et à Rategno de l'exploiter au mieux pour leur construction logique; cela leur garantit de ne pas entrer ouvertement en conflit avec le droit ecclésiastique, ce qui aurait pu déclencher une réaction de l'Église (ibid.: 343). Pourtant, se limiter à ces argumentations n'est pas suffisant.

Tout démonologue qui souhaite se pencher sur la question de la réalité du vol et des pratiques des sorciers et des sorcières doit effectivement repérer d'autres arguments pour pouvoir légitimer ultérieurement son propos. Le procédé de Bernard de Côme et d'autres démonologues est alors le plus traditionnel possible. En effet, ils cherchent à repérer toutes les références bibliques et patristiques qui pourraient corroborer leur discours. Par exemple, l'épisode de Jésus transporté par Satan sur le pinacle du Temple de Jérusalem (Mt 4,5) est largement utilisé. Ou encore lorsque Philipe, dans le livre des Actes des Apôtres, est enlevé et conduit par le Saint-Esprit jusqu'à Azoth (Ac 8, 39-40). De plus, la capacité des démons à déplacer des corps a été largement théorisée par Augustin dans sa Cité de Dieu (426). L'évêque d'Hippone y développe l'idée que les démons peuvent transporter des entités physiques (XVIII, 18,2) et donc des êtres humains.

27 Augustin, dans les pages de son œuvre, donne une autre précision qui sera fondamentale dans le développement des théories démonologiques qui amèneront à la création du crime de la sorcellerie diabolique. En effet, il expose que :

Ce qu'il faut croire très fermement, c'est que Dieu tout-puissant peut faire tout ce qu'il veut, soit pour châtier, soit pour aider l'homme; et que les démons, ces créatures angéliques mais perverties par un vice volontaire, ne peuvent exécuter quoi que ce soit par la puissance de leur nature, sauf ce que permet Dieu, dont bien des jugements sont cachés, mais dont aucun n'est injuste. (XVIII, 18, 2) 

démons, largement reprises par Rategno et d'autres démonologues, tels que Nicolas Jaquier dans son Flagellum hereticorum fascinariorum (1458), permettent d'éviter le piège dualiste. Ce dernier voit s'opposer le Bien au Mal dans une lutte constante, à l'instar des deux entités indépendantes l'une de l'autre (Belli \& Estuardo Flaction, 2019 : 344-346).

\section{Le rôle des rêves}

La démarche de Rategno pour démontrer la réalité des pratiques de la secte des striges n'est pas encore achevée. Sur le modèle de Girolamo Visconti (Belli \& Estuardo Flaction, 2019 : 180), l'inquisiteur de Côme fait un pas ultérieur qui semblerait annuler le débat. Rategno concède - "posito sed non concess ${ }^{26}$ » - que les striges pourraient participer aux assemblées diaboliques et y pratiquer tous les maléfices en rêve ou par le biais d'une illusion diabolique. Cependant, et l'inquisiteur de Côme l'explicite clairement, ces individus pestiférés, « une fois réveillés et ayant repris le contrôle de leurs sens, se seraient félicités des actions commises lors du rêve [...], en les considérant comme parfaitement réelles. Pour ces raisons, elles seraient vraiment des apostats, idolâtres et hérétiques » (ibid. : 364-365). Rategno doit alors pouvoir argumenter que le plaisir pour un péché ressenti lors d'un rêve est punissable de la même manière qu'un péché commis en état de veille. Pour ce faire, il se base sur les propos de Thomas d'Aquin sur les pollutions nocturnes. Dans son Commentaire des Sentences (1254-1256), l'auteur expose que si celles-ci surviennent durant le sommeil, elles sont de simples péchés véniels; toutefois, elles sont considérées comme des péchés mortels si l'homme en tire du plaisir dans l'avenir (lib. IV, d.9, q. 1, a.4, q. c. 1, ad. 5). Il en va de même pour les rêves hérétiques (Belli \& Estuardo Flaction, 2019 : 365). D'ailleurs, comme l'a écrit Augustin d'Hippone dans la Cité de Dieu : «se plaire à des crimes supposés est un crime véritable » (XVIII, 12, 779).

Afin de résumer ce bref aperçu, nous pouvons affirmer que le procédé logique proposé par Bernard de Côme pour démontrer la réalité du ludus n'est absolument pas nouveau. Notre inquisiteur s'inscrit parfaitement dans le débat démonologique de la fin $\mathrm{du}_{\mathrm{Xv}} \mathrm{x}^{\mathrm{e}}$ siècle, en reprenant les mêmes éléments d'argumentation que nous pouvons retrouver dans plusieurs traités antérieurs aux De Strigiis. Dès lors, pourquoi alors Rategno a-t-il eu besoin de composer son œuvre? La réponse est très probablement à rechercher dans sa nécessité de pouvoir légitimer son entreprise inquisitoriale dans le diocèse de Côme. Il va de soi qu'il a très probablement prononcé des condamnations. Rategno doit donc les justifier, en démontrant que ces hommes et ces femmes sont vraiment des apostats, idolâtres et hérétiques, qui se rendent réellement aux assemblées diaboliques et que la peine de mort est alors légitime (Belli \& Estuardo Flaction, 2019 : 367).

\section{Les striges : véritables apostats, idolâtres et hérétiques}

31 Rategno s'applique largement tout au long de son traité à définir ces personnes comme apostats (apostatce), idolâtres (idolatræe) et hérétiques (høeraeticœ) (Belli \& Estuardo Flaction, $2019: 280$ ). L'utilisation de ces trois concepts pour caractériser les striges n'est pas anodin et peut légitimer encore une fois la mise à mort. D'ailleurs, le seul crime 
d'hérésie accorderait à l'accusé - s'il n'est pas relaps - la possibilité de se repentir, en retournant ainsi au sein de l'Église. Rategno l'affirme sans cesse, comme beaucoup d'autres avant lui ${ }^{27}$, les crimes des striges ne sont pas uniquement du domaine de l'hérésie. Bien aucontraire, ils découlent de quelque chose de nettement pire: l'accumulation des accusations hérétiques, avec celles d'idolâtres et d'apostats. Les trois commises en pleine conscience. Cela dit, cette surqualification pousse, sans possibilité d'appel, le coupable au bûcher (Ostorero, $2010: 32$; Belli \& Estuardo Flaction, 2019: 376). D'ailleurs, souligner sans cesse ces trois accusations - les trois qui découlent du milieu ecclésiastique - permet à Bernard de Côme de faire de la chasse aux sorcières une prérogative exclusivement inquisitoriale.

La question centrale que l'inquisiteur de Côme se pose maintenant est de savoir comment repérer les membres de la secte des striges et de quelle manière les punir.

Le premier pas est d'affirmer que les interrogatoires des témoins et les dénonciations sont certainement fondamentales, mais pas suffisantes (Belli \& Estuardo Flaction, 2019 : 284). L'enquêteur doit prêter une attention particulière à cette étape de la procédure. Jamais, affirme Rategno, il ne faudra demander aux suspects s'ils ont vu une personne précise. Il faut privilégier les questions plus larges, en exhortant ceux qui sont interrogés à être les plus honnêtes possibles. D’ailleurs, lors des assemblées, les démons auraient la capacité de prendre l'aspect d'un individu complétement innocent ${ }^{28}$. Il faut alors repérer les moyens pour enquêter de la manière la plus fiable possible. Un stratagème proposé par Rategno serait alors d'aller chercher là où de possibles maléfices ont lieu. Ces derniers font partie des typologies typiques des écrits démonologiques. Il s'agit notamment des crimes qui visent la santé des êtres humains et des animaux, tout comme ceux qui portent atteinte à la nature et aux récoltes (ibid. : 286).

34 L'inquisiteur de Côme explique alors qu'il faudrait enquêter par exemple dans les cas d'infertilité. Les maléfices pourraient être les responsables du « refroidissement » des hommes, en les rendant par conséquent impuissants ${ }^{29}$; ou encore, le diable aurait la capacité de manipuler l'appréciation et l'imagination de l'homme, de sorte à rendre la femme dégoûtante ${ }^{30}$. De plus, une menace faite lors d'une bagarre pourrait cacher une mauvaise intention de la part d'une strige. Ce sont ces épisodes qui doivent attirer l'attention des inquisiteurs. Des enfants qui tombent malades, le bétail qui meurt soudainement ou, encore, une tempête de grêle qui ravage toute la récolte. D'après Rategno, c'est derrière ces tragédies que la secte des striges pourrait se cacher. Or, il faut néanmoins souligner un aspect particulier. Dans toutes les pages du De Strigiis, il n'est jamais question de vampirisme, cannibalisme ou infanticide. Comme nous le savons, ces accusations sont à la base du crime de sorcellerie diabolique, au nord et au sud des Alpes ${ }^{31}$. Pourtant, Rategno ne fait jamais mention de ces crimes. Comment expliquer ce fait? Nous pouvons estimer que, dans une optique de conflits de juridiction, parler de meurtres, surtout si des enfants sont impliqués, aurait éveillé l'intérêt des tribunaux séculiers. Chose que l'inquisiteur de Côme souhaitait éviter.

\section{Conclusion}

Le De Strigiis est l'exemple d'un traité particulièrement modéré et pragmatique: Bernard Rategno cherche à poser des bases juridiques et théologico-philosophiques qui soient les plus solides possibles afin de pouvoir mener correctement la chasse aux 
sorcières. Son œuvre est alors pensée pour ses collègues inquisiteurs. S'il a eu besoin de produire un tel approfondissement sur les striges qui peuplaient son territoire de juridiction, c'est à cause de la nécessité de donner une légitimation à son action de chasseur de sorciers et de sorcières: le traité doit démontrer que ces individus ont vraiment et réellement commis les choses pour lesquelles on les poursuit. La très longue dissertation sur la réalité du vol, que nous avons juste effleurée, et par conséquent du ludus, sert justement ce but précis.

En plus de cela, Bernard de Côme pense à délimiter clairement la juridiction de l'Église face aux tribunaux laïcs : les striges découlent en effet uniquement de la compétence des inquisiteurs. Pour ce faire, il les définit comme apostats, idolâtres et hérétiques. Nous pourrions ainsi estimer que la proposition étymologique de Rategno - assimilation strige/Styx - sert à rendre ces personnes les plus infernales possibles. En ignorant l'étymologie classique de la "strix », Rategno semble refuser d'assimiler la secta strigiarum uniquement à l'univers féminin. Il délaisse ainsi le côté sexuel (strixfemme), pour se concentrer sur la dimension infernale de ces êtres, hommes ou femmes. Cette dimension serait de la compétence unique de la justice ecclésiastique. Le caractère si mitigé du De Strigiis, ainsi que ses conseils procéduraux ont sûrement attiré l'attention de Francisco Peña. Le De Strigiis a en effet été réédité et commenté environ un demi-siècle après sa rédaction par le canoniste aragonais. Cette démarche est certainement le résultat d'une volonté de la part de la papauté de renouveler le droit inquisitorial et de donner à la nouvelle génération d'inquisiteurs de la fin $\mathrm{du} \mathrm{XVI}^{\mathrm{e}}$ siècle les traités les plus appropriés pour accomplir sa tâche.

\section{BIBLIOGRAPHIE}

Ammann-Doubliez Chantal (2020), Procès de sorcellerie dans la vallée de Conches (1466-1467) et chasses aux sorciers et sorcières en Valais au Xve siècle = Hexenprozesse im Goms (1466-1467) und Hexenverfolgungen im Wallis im 15. Jahrhundert: Kurzfassung, Sion : Archives de l'État du Valais. ANDENMATTEN Bernard (à paraître), « Geste de soumission, formalité bureaucratique ou rituel curial ? L'hommage vassalique dans l'État princier savoyard du bas Moyen Âge ", E. Pibiri et al. (org.), Les gestes à la cour (XII ${ }^{e}$-XVII ${ }^{e}$ siècle). Colloque international (Université de Lausanne, 2018), Florence : SISMEL - Edizioni del Galluzzo.

Augustin (1959), La cité de Dieu (De civitate Dei), B. Dombart, G. Bardy \& G. Combès (éd.), Paris : Desclée.

Belli Alessia \& EstUARDo Flaction Astrid (2019), Les striges en Italie du Nord :édition critique et commentaire des traités de démonologie et sorcellerie de Girolamo Visconti (Milan, c. 1460) et de Bernard Rategno (Côme, c. 1510), Florence : SISMEL - Edizioni del Galluzzo.

DUNI Matteo (2013a), « Un manuale inedito per cacciatori di streghe: il Formularium pro exequendo Inquisitionis officio di Modesto Scrofeo (c. 1523) », Archivio storico italiano, 171(2), 339-358. 
DUNI Matteo (2013b), « The Editor as Inquisitor: Francisco Peña and the Question of Witchcraft », M. Israëls \& L. A. Waldman (éds), Renaissance Studies in Honor of Joseph Connors, Florence : The Harvard University Center for Italian Renaissance Studies, 306-312.

GIORGETTA Giovanni (2003), « I processi dell'Inquisizione in Valtellina e Valchiavenna nel XV secolo: dalla "buona società" alla stregoneria ", Streghe, diavoli e Sibille: atti del convegno Como (18-19 maggio 2001), Côme : Nodolibri, 21-31.

GIORGETTA Giovanni (2007), « Inquisitori e giudici laici nel territorio dell'antica Diocesi di Como », Caccia alle streghe in Italia tra XIV e XVII secolo: atti del IV Convegno nazionale di studi storico antropologici (Triora (Imperia), 22-24 ottobre 2004), Bolzano : Praxis 3, 97-126.

GURY Françoise (1998), « À propos de l'image des incubes latins », Mélanges de l'École française de Rome. Antiquité, 110(2), 995-1021.

HANSEN Joseph (1963), Quellen und Untersuchungen zur Geschichte des Hexenwahns und der Hexenverfolgung im Mittelalter; mit einer Untersuchung der Geschichte des Wortes Hexe von Franck (ouvrage original publié en 1901), Hildesheim : G. Olms.

HITZ Florian (2018), « Grisons », Dictionnaire historique de la Suisse, <https://hls-dhs-dss.ch/fr/ articles/007391/2018-01-11/>.

LAVENIA Vincenzo (2016), « Rategno, Bernardo », Dizionario biografico degli Italiani (vol. 86), Rome : Istituto dell'Enciclopedia italiana, 554-556.

OSTORERo Martine (2010), «Crimes et sanctions dans la répression de la sorcellerie à la fin du Moyen Âge », Revue historique vaudoise, 118, 17-33.

OSTORERo Martine (2011), Le diable au sabbat. Littérature démonologique et sorcellerie (1440-1460), Florence : SISMEL - Edizioni del Galluzzo.

Ostorero Martine, Paravicini Bagliani Agostino, UtZ Tremp Kathrin \& ChÈne Catherine (dir. \& collab.) (1999), L'imaginaire du sabbat. Édition critique des textes les plus anciens (1430 c.-1440 c.), Lausanne : Université de Lausanne.

OSTORERo Martine, UTZ TREMP Kathrin \& MODESTIN Georg (dir. \& collab.) (2007), Inquisition et sorcellerie en Suisse romande. Le registre AC 29 des Archives cantonales vaudoises (1438-1528), Lausanne : Université de Lausanne.

TAVuZZI Michael (2007), Renaissance Inquisitors. Dominican Inquisitors and Inquisitorial Districts in Northern Italy, 1474-1527, Leiden : Brill.

THÉRY Julien (2013), « A Heresy of State. Philip the Fair, the Trial of the "Perfidious Templars”, and the Pontificalization of the French Monarchy ", Journal of Medieval Religious Cultures, 39(2), 117-148.

VAN DER LUGT Maaike (2004), Le ver, le démon et la Vierge. Les théories médiévales de la génération extraordinaire, Paris : Les Belles Lettres.

\section{NOTES}

1. Cette contribution reprend, avec quelques modifications et mises à jour bibliographiques, la publication suivante : Belli \& Estuardo Flaction (2019).

2. Pérouse, 19 mai 1478 : «Bernardus de Cumis, qui per capitulum generale Romanum (1474) fuerat assignatus ad legendum Sententias in Sacro palatio, habuit licentiam, quod possit legere in quacunque 
universitate pro forma et gradu» (Rome, AGOP, IV, 3 fol.372, cité dans Hansen, 1963 [1901]: 279-280).

3. 21 mai 1505 : « fr. Bernardus de Como instituitur inquisitor Cumanus » (Rome, AGOP, IV, 17 fol. 172, cité dans Hansen, 1963 [1901] : 280).

4. Cette république est composée par la Ligue de la Maison-Dieu (1367), la Ligue Grise (1395/1424) et la Ligue des Dix-Juridictions (1436). Aujourd'hui elle correspond au canton suisse des Grisons, ce territoire est situé au sud-est de la Suisse, limitrophe avec la Valteline et la Valchiavenna (Hitz, 2018).

5. 30 août 1506 : «Specialiter, ad nomine et vice ipsius communis Clavenne, interveniendum omnibus et quibuscumque actis et processibus necessariis et expendiendis, faciendis et exercendis per reverendum dominum fratrem Bernardum de Retegno, priorem conventus ordinis predicatorum Sancti Iohannis extra muros Comi et inquisitorem heretice pravitatis, contra quoscumque hereticos et inculpatos ac infamatos de crimine heresis. » (ASSo, Fondo notarile, vol. 557, cité dans Giorgetta, 2003 : 28)

6. Pour une édition scientifique du Tractatus de Strigiis, voir Belli \& Estuardo Flaction (2019: 265-298).

7. "Secta striarum seu strigiarum qualiter pertinet ad inquisitores procedere contra eos, eo quia sunt haereticae, quia apostatae, et qualiter possunt ire corporaliter ad earum ludum, et quomodo possunt deprehendi illi, qui sunt de illa secta, ponam in fine totius huius operis, ubi diffuse de his omnibus tractabo, et etiam ponam aliqua ad declarationem capituli Episcopi 26, qucestione 5. " (Lucerna inquisitorum hereticce pravitatis R.P.F. Bernardi Comensis ordinis praedicatorum, ac Inquisitoris egregii, in qua summatim continentur quicquid desideratur ad huiusce Inquisitionis sanctum munus exequendum Mediolani, apud Valerium et Hieronymum fratres Metios, 1566, fol.71r-v, cité dans Belli \& Estuardo Flaction, $2019: 7)$

8. Pour plus d'informations, voir Belli \& Estuardo Flaction (2019: 253-254).

9. "Secta qucedam abomniabilis virorum videlicet, et procipue mulierum, a pluribus annis citra in partibus Italice, satore omnium malorum diabolo procurante, damnabiliter insurrexit, quas alii maschas appellant, nos autem in Lombardia strigias nuncupamus, a stix, Stigis, vocabulo infernum seu paludem infernalem significante, quia tales personce diabolica sunt et infernales, vel a Stigetos Graece, quod est tristitia latine, eo quia plurimos faciant tristes maleficiis suis. » (De Strigiis, cité dans Belli \& Estuardo Flaction, $2019: 267$ )

10. Nous pouvons citer les cas de Pierre Chavaz (Champvent, 1448), Pierre Antoine (Ouchy, 1449) et Jean Massot (Dommartin, 1525) : voir Ostorero, Utz Tremp \& Modestin (2007).

11. Alphonse de Castro, De iusta haereticorum punitione, libri tres, I, cap. XVI, fol. 85r : "Strix autem avis qucedem est nocturna, et importuna sic dicta a stridore, quem facit. De hac sic dicit Lucanus libro sexto »; Alphonse de Castro fait référence au $6^{\mathrm{e}}$ livre de La Pharsale de Lucain (39-65), où il est écrit, aux v. 689-690, «[...] quod strix nocturna queruntur, quod strident ululantque ferce, quod sibilat anguis». Diego de Simancas, De catholicis institutionibus liber, tit. XXXVII, De lamiis, § 2, p. 278 : "Stryx a stridore vocis dicta, avis est nocturna et importuna: inde dicuntur stryges mulieres istce, quia noctu praecipue versantur in maleficiis, et infantium sanguinem sugunt. Nam ut Festus aiunt [...]. » (Belli \& Estuardo Flaction, 2019 : 302)

12. Pour une analyse plus détaillée sur l'utilisation du terme «strige», voir Belli \& Estuardo Flaction (2019: 35-41).

13. «Hæe personce congregantur in certis locis per oppida et villas certis temporibus, procipue in nocte diem Veneris prcecedente, apparente eis doemone in forma humana visibili, et quando in principio de illa secta esse volunt seu incipiunt, primo et ante omnia coram ipso diabolo ad iussum eius abnegant sanctam fidem et sanctum baptisma, ac etiam Dominum Deum et Beatam Virginem Mariam, et postea conculcant crucem aliquam ibi ab una illarum strigiarum actam in terra. Quibus omnibus peractis, faciunt fidelitatem in manibus ipsius diaboli, acceptantes eum in dominum suum, et promittunt ei in omnibus se velle semper obedire, et in signum horum omnium tangunt ei manum cum ipsarum manu sinistra post earum tergum versa, offerentes eidem diabolo aliquid in signum subiectionis, et ita abinde citra semper reputant ipsum 
diabolum esse verum earum Dominum et Deum suum. Deinde quotiescunque vadunt ad illam congregationem, quam ludum bonce societatis appellant, semper faciunt reverentiam ipsi diabolo in forma humana apparenti, et ipsum capite inclinato profunde adorant ut verum Deum suum [...]. » (De Strigiis, cité dans Belli \& Estuardo Flaction, 2019 : 267-268)

14. Il suffit de penser au procès contre les Templiers (1307), qui étaient accusés d'avoir renié le Christ et d'avoir craché sur la croix (Théry, $2013: 123$ ).

15. «Et en effet le dit jeune homme, capturé dans la juridiction des Bernois avec sa femme [...] déclara: "[...] Le rituel par lequel je fus séduit, dit-il, est le suivant. Il faut d'abord que le dimanche, avant que l'eau bénite ne soit consacrée, le futur disciple entre dans l'église avec les maîtres et qu'il abjure devant eux le Christ, sa foi, le baptême et l'Église Universelle. Ensuite il prête hommage au magisterulus [...] c'est en effet de cette manière [...] qu'ils appellent le démon." " (Ostorero, Paravicini Bagliani, Utz Tremp \& Chène, 1999 : 157)

16. Nous faisons ici référence aux traités Lamiarum sive striarum opusculum et Opusculum de striis. Pour l'édition scientifique et l'analyse, voir Belli \& Estuardo Flaction (2019 : 306-314).

17. Pour plus d'informations, voir Andenmatten (à paraître).

18. "[...] ac alia plura spurcissima perpetrant cum ipso diabolo, eis in specie humana apparente, et se viris succumbum, mulieribus autem incubum exhibente, ac, ut omnimodam delectationem veneream eis impendant, ad omnem prorus earum appetitum se per omnia coaptante. " (De Strigiis, cité dans Belli \& Estuardo Flaction, 2019 : 268) Pour plus d'informations sur ces figures de la mythologie romaine, voir Gury (1998) et Van der Lugt (2004).

19. "Ad quam congregationem seu ludum prefatce pestiferce personce vadunt corporaliter et vigilantes, ac in propriis earum sensibus. » (De Strigiis, cité dans Belli \& Estuardo Flaction, 2019 : 268)

20. Dans l'état actuel de la recherche, nous n'avons aucune trace de ces affaires juridiques.

21. "Unum est quod omnes huius pestiferce secte, sive mares sive fœemince, quasi uno ore et una lingua fatentur, se eundem modum per omnia et uniformiter observare ubique locorum in abnegando fidem [...] quae in tali ludo seu congregatione fieri contingunt, ita quod omnes in idem conveniunt et semper convenerunt a principio illius sectee usque in presens tempus, ut ex earum omnium confessionibus ubique locorum per totam Italiam in manibus inquisitorum nostrorum factis, et scriptis in processibus contra eas formatis, liquide constat; quod esse non posset, et non ita in omnibus se conformarent, si fantastice et in somniis eis ista contingerent, precipue cum fantasmata et somnia secundum varietatem causarum, et qualitatem personarum, pro loco et tempore varientur.» (De Strigiis, cité dans Belli \& Estuardo Flaction, 2019 : 268)

22. " Aliud argumentum est, quod interdum contigit, quasdam personas deferri per diabolum ad aliquem locum distantem, ubi talis ludus seu congregatio strigiarum fiebat, et dum essent in via eundo vel redeundo, Deo volente, dimisse sunt, et inventce extra patriam suam et extra terras suas, quod esse minime potuisset si prcedicta eis fanstatice contigissent, aut se deferri somniassent. » (De Strigiis, cité dans Belli \& Estuardo Flaction, $2019: 271)$

23. « Preetera plurimce personce huius perfidee sectee, transactis iam plurimis temporibus, per inquisitores hoeretica pravitatis fuerunt tradite brachio seculari, exigentibus id demeritis suis, et conbustee, quod minime factum fuisset, neque summi pontifices hoc tolerassent, si talia tantummodo fantastice et in somniis contingerent, et tales personce realiter et veraciter horetice non essent, et in horesi realiter et manifeste deprehensa. Nam Ecclesia non punit crimina nisi sint manifesta et vere deprehensa, distinctione 32, capitulo Erubescant ; causa 32, quaestione 5, [ capitulo 23] Christiana; causa 2, quaestione 5, [capitulo 20] Consuluisti et [causa] 6, quastione 1, [capitulo 7] Non omnia. » (De Strigiis, cité dans Belli \& Estuardo Flaction, 2019 : 271)

24. "Sed ecce contra hrec, quee iam dicta sunt, quidam insurgunt, et prcedictam veritatem destruere satagentes, dogmatizando affirmant, quod huiusmodi strigice nullo modo vadant ad illum ludum corporaliter et realiter, sed quod fantastice, ac illusorie, et in somniis eis omnia prodicta contingant, et in huius opinionis suce fomentum adducunt textum capitulum Episcopi 26, quæestione 5. » (De Strigiis, cité dans Belli \& Estuardo Flaction, 2019 : 273) 
25. «[...] quia si quis bene considerat sensum canonis illius, videbit clare quod non negat quin sit possibile tales personas pedester, et corporaliter ire ad predictum suum ludum, sed necque negat, quod sit possibile, easdem deferri vere, realiter, et corporaliter, a diabolo de loco ad locum [...]. » (De Strigiis, cité dans Belli \& Estuardo Flaction, 2019 : 274)

26. " Postremo, ut adversariorum ora ex toto concludam, posito sed non concesso, quod, ut adversarii ipsi affirmant, istce strigice nullo modo vadant corporaliter ad ludum, et quod nunquam realiter ac veraciter vigilantes abnegaverint fidem [...]; tamen ex quo postea, quando sunt in vigilia et in propriis sensibus constitutee, credunt et firmiter tenent se praedicta fecisse et habent ea rata et firma, ac ea confirmant, et contentee sunt quod sibi talia acciderint, ac eis omnibus consentiunt cum quadam animi complacentia, credentes firmiter se fidem catholicam abnegasse, et dcemonem pro suo vero deo habere [...]. Liquido et clarius luce meridiana constat, quod sunt verce et proprice apostate, idolatrce et haereticce. " (De Strigiis, cité dans Belli \& Estuardo Flaction, 2019 : 278)

27. Nous faisons ici surtout référence au traité Ut magorum et maleficiorum errores... de Claude Tholosan (c. 1436), juge mage du Dauphiné. Il s'agit du premier effort d'une explication doctrinale de ces trois accusations. Nous pouvons également citer le traité Flagellum hereticorum fascinorum (1458) du dominicain Nicolas Jacquier.

28. "Advertas insuper, et sis bene cautus, ne de facili facias aliquam detineri propter inculpationes talium strigiarum tantum, quia posset contingere quod dcemon assumeret personam alicuius, et se sub forma illius se prcesentaret in ipso ludo, ut illam personam infamet, et tamen illa persona erit innocens, et de tali crimine nullo modo culpabilis. » (Belli \& Estuardo Flaction, 2019 : 284)

29. «Alio modo, quia potest refrigidare hominem ab actu illo, adhibendo occulte virtutes rerum, quas optime novit ad hoc validas. » (Belli \& Estuardo Flaction, 2019 : 287)

30. "Tertio modo turbando extimationem et imaginationem, qua reddit mulierem exosam, quia postet in ipsa imaginatio imprimere. » (Belli \& Estuardo Flaction, 2019 : 287)

31. Nous faisons ici référence aux cinq premiers traités de l'Imaginaire du sabbat (Ostorero, Paravicini Bagliani, Utz Tremp \& Chène, 1999) et, entre autres, aux œuvres de Girolamo Visconti de la moitié du $\mathrm{Xv}^{\mathrm{e}}$ siècle.

\section{RÉSUMÉS}

$\mathrm{Au}$ tout début $\mathrm{du} \mathrm{XVI}^{\mathrm{e}}$ siècle, Bernard Rategno, inquisiteur dominicain de Côme, rédige le Tractatus de Strigiis. Ce petit traité de démonologie fait état de la croyance selon laquelle une secte d'hommes et de femmes (les striges du titre), se rendrait de nuit au «jeu» (ludus) pour se soumettre au diable et pour pratiquer des actions néfastes telles que le blasphème, les orgies sexuelles et les maléfices. Dans les pages de son opuscule, Rategno se questionne alors sur la réalité de ces pratiques. Est-ce que ces individus pestiférés se rendent vraiment aux assemblées diaboliques? Ou est-ce que le ludus et les actes commis ne sont rien d'autre qu'une illusion provoquée par le diable? Quelle est la place du célèbre canon Episcopi dans ces raisonnements? L'œuvre de l'inquisiteur de Côme s'inscrit ainsi parfaitement dans le débat démonologique - du nord et du sud des Alpes de la fin du XIV siècle - qui cherche sans cesse à répondre à ces interrogations.

In the early 16th century, Bernard Rategno, a Dominican inquisitor in Como, wrote the Tractatus de Strigiis. This little treatise on demonology mentions the belief that a sect of men and women (the striges of the title) would go at night to the "game" (ludus) to submit to the devil and to 
practice evil deeds such as blasphemy, sexual orgies and curses. In the pages of his opuscule, Rategno then questions the reality of these practices. Do these pestiferous individuals really go to the diabolical assemblies? Or are the ludus and the acts committed nothing more than an illusion provoked by the devil? Where does the famous canon Episcopi fit into these arguments? The work of the Inquisitor of Como thus fits perfectly into the demonological debate-both north and south of the Alps at the end of the 14th century-which constantly seeks answers to these questions.

\section{INDEX}

Keywords : Alps, canon Episcopi, Como, goddess Diana, demonology, duchy of Milan, Medieval Inquisition, Italy, Lombardy, manual for inquisitors, Order of Preachers (Dominican Order), Witchcraft, nocturnal flight

Mots-clés : Alpes, canon Episcopi, Côme, déesse Diane, démonologie, duché de Milan, Inquisition médiévale, Italie, Lombardie, manuel d'inquisiteurs, ordre des Prêcheurs (ordre des Dominicains), sorcellerie, vol nocturne

\section{AUTEUR}

\section{ALESSIA BELLI}

Université de Lausanne 